\title{
Urinary Microbiome
}

\section{Huseyin Ozgur Kazan, MD and Bulent Erol, MD*}

Department of Urology, Faculty of Medicine, Istanbul Medeniyet University, Turkey

*Corresponding author: Bulent Erol, M.D, Department of Urology, Faculty of Medicine, Istanbul Medeniyet University, Turkey, Tel: 00905323526564

\begin{abstract}
Small microorganisms are colonised in various sites of human body including urinary tract. Although they could not be determined by standard culture techniques, up-to-date it has been suspected that urinary system is not out of any microorganisms. Simply, total of these microorganisms form urinary microbiome. Contribution to well being of tract or cause of diseases and which microorganisms play the role are questions to be answered. Bladder cancer recurrence, prostate cancer ethiology, relationship with renal Stones, chronic pelvic pain, bladder pain, urinary incontinence are most studied subjects about urinary microbiome to detect targeted therapies, explore protective therapeutic strategies or find new biomarkers.
\end{abstract}

\section{Introduction}

Human body includes 10 times more microbial cells than human cells and most of them can't be specified by standard culture technics. These microbial cells are encoded in the human genome, having great impact on various diseases or well-being of different organ systems. To understand microbiome researchs, first some terms must be clarified. Microbiota is the total of microorganisms in special organs, which genome forms microbiome. Genetics, dietary, environmental influence, hosts immune function, neurotransmitters and various metabolites play important role forming special microbiomes. Most of the studies maintain that the development of the microbiome starts at birth. Alterations of microbiome between cesarean and vaginal births had been seen in various researchs. Gut microbiota of breast milk fed neonatals include dominantly bifidobacteria, on the other hand formula fed neonatals microbiota include bacteriodes fragilis, enterobacteriae, clostridium difficile. Maternal infections, diseases, hospitalization, antibiotics use are other factors which have influence in microbiome [1-3].

Human microbiome Project (HMP) was started by National Institute of Health in 2008 to determine all the microbial genome sequences and to define its role in someones health and disease. From 5 different sites of human body, samples were studied: nasal passages, oral cavity, skin, gastrointestinal tract, and urogenital tract. The HMP is extracting $16 \mathrm{~S}$ rRNA and making metagenomic sequencing to determine microbiome. 16S rRNA is like a fingerprint for bacterials metogenomes. Therefore $16 \mathrm{~S}$ rRNA is the most specific part of bacterial genom, which is used in protein synthesis [4].

\section{Methodology Studying Urine Samples}

To study urine samples $16 \mathrm{~S}$ rRNA extraction is the most common way. The Ibis T-5000 Universal Biosensor technoloy, which uses PCR, electrospray ionization (ESI), time of flight (TOF) and mass spectrometer (MS) in general.

First DNAs were amplified by the polymerase chain reaction (PCR). The amplicons revealed by PCR were weighed by electrospray ionization (ESI), time of flight (TOF) and mass spectrometer (MS) to determine the molecular mass. Every molecular mass consists of a combination of four nucleotides $(A, C, G, T)$. The genomic species were identified through the database, which containes bacterial, fungal species sequences.

In laboratory phase all urine samples were divided to $3 \mathrm{ml}$ parts and centrifuged at $10000 \mathrm{rpm}$ by $3 \mathrm{~min}$ utes, supernatant was removed and the pellets nucleic acid sample was revealed by Tissues kits. Then each sample was amplified by PCR. 
$16 \mathrm{~S}$ rRNA sequencing is not the only way to study microbiome; DNA extraction, RNA extraction and extraction of protein or small molecules are options.

\section{The Microbiota of the Urinary Tract}

Relationship between microbiome and urological diseases are studied in a wide range of specialities: Urolithiazis, interstitial cystitis, chronic pelvic pain syndrome, incontinence, urinary tract infections and urooncology.

Several studies pointed urinary and gut microbiome diversities of chronic prostatitis/chronic pelvic pain syndrome patients compared with controls. In the gut microbiome diversity has been stated less in CP/CPPS patients and distribution was different than controls [5]. In the same study lower counts of Prevotella were also a remarkable end-point, which may be used as a biomarker. In a similar study urinary microbiome of CP/ CPPS patients have higher diversity than control group. The prevalance of anaerob bacteries was significantly higher in CP/CPPS group [6]. In another study by Horwitz, et al. it has been shown that microbial diversity plays a protective role against invasive urinary tract infections of the catheterized bladders [7].

Stool microbiota was also studied to detect significant species, potential biomarkers and targeted therapies for Interstitial cystitis/bladder pain syndrome patients. Quantitative PCR of stool DNA has revealed some deficient species like $E$. sinensis, $C$. aerofaciens, $F$. prausnitzii, $O$. splanchnicus, and $L$. longoviformis. These deficient species might be used as biomarkers in stool samples. Furthermore metabolic pathways were determined by the same study, as glyceraldehyde levels were increase in IC patients. It might play an important role for targeted therapy [8].

In various studies the occurence of Stone disease and relationship with Oxalobacter formigenes were researched. Recent studies showed that lower prevalance of this bacteria is associated with increased urinary oxalate concentration [9]. Siener, et al. described that $O x$ alobacter formigenes decreased the urinary oxalate by reducing the intestinal absorbtion.

Inflammation of prostate and its atrophy, high grade prostate intraepithelial neoplasia are hypothesized having great impact on prostate cancer pathophysiology. Genetic polymorphisms seen at cellular inflammation are modelling the baseline of the hypothesis. Cytokines like IL-6, IL-8 are responsable for prostate cancer development [10]. Some studies have also shown that history of sexually transmitted diseases are increasing the probability to develop prostate cancer [11-13]. In that case anti-inflammatory drug use might prevent the early prostate cancer development.

Nowadays probiotic use is very common, which made specific development against various dieases. Probiotics show their effects by reducing mucosal inflammation through inhibition of the NF-KB pathway and IL-6,-8 [14]. In a double blind placebo-controlled randomized trial it has been revealed that $L$. casei reduced the prevalance of superficial bladder cancer recurrence [15]. Superiority to BCG treatment is controversial and couldn't make any statement.

Womens chronic pelvic pain syndrome is another challenging subject of urology, because of its difficulties in diagnosis and treatment [16-18]. Nickel, et al. Compared microbiomes of women with chronic pelvic pain flare and non flare groups. Overall 81 species were specified first voided urine (VB-1) and 73 species mid stream urine (VB-2). Candida and Saccharomyces prevalance were significantly higher in flare group and especially in VB-2. A bladder involvement through that result is a great possibility. For planning targeted therapies that might be a baseline [16].

\section{Conclusion}

Urinary microbiome is researched almost in all fields of urology. To develop specific biomarkers, better diagnostic evaluation, targeted treatment and protection against diseases these researchs are promising. The use of probiotics against recurrent urinary tract infections, targeted therapies against funghi (Candida and Saccharomyces), the role of Provetella are ready to explore.

\section{References}

1. Cho I, Blaser MJ (2012) The human microbiome: At the interface of health and disease. Nat Rev Genet 13: 260-270.

2. Ursell LK, Metcalf JL, Parfrey LW, Knight R (2012) Defining the human microbiome. Nutr Rev 70: S38-S44.

3. Lazarevic V, Whiteson K, Huse S, Hernandez D, Farinelli L, et al. (2009) Metagenomic study of the oral microbiota by Illumina high-throughput sequencing. J Microbiol Methods 79: $266-271$

4. NIH HMP Working Group, Peterson J, Garges S, Giovanni M, Mclnnes P, et al. (2009) The NIH human microbiome project. Genome Res 19: 2317-2323.

5. Shoskes DA, Wang H, Polackwich AS, Tucky B, Altemus J, et al. (2016) Analysis of gut microbiome reveals significant differences between men with chronic prostatitis/chronic pelvic pain syndrome and controls. J Urol 196: 435-441.

6. Shoskes DA, Altemus J, Polackwich AS, Tucky B, Wang $\mathrm{H}$, et al. (2016) The urinary microbiome differs significantly between patients with chronic prostatitis/chronic pelvic pain syndrome and controls as well as between patients with different clinical phenotypes. Urology 92: 26-32.

7. Horwitz D, McCue T, Mapes AC, Ajami NJ, Petrosino JF, et al. (2015) Decreased microbiota diversity associated with urinary tract infection in a trial of bacterial interference. $J$ Infect 71: 358-367.

8. Braundmeier-Fleming A, Russell NT, Yang W, Nas MY, Yaggie RE, et al. (2016) Stool-based biomarkers of interstitial cystitis/bladder pain syndrome. Sci Rep 18: 26083.

9. Siener R, Bangen U, Sidhu H, Hönow R, von Unruh G, et al. (2013) The role of Oxalobacter formigenes colonization in calcium oxalate stone disease. Kidney Int 83: 1144-1149. 
10. Puhr M, De Marzo A, Isaacs W, Lucia MS, Sfanos K, et al (2016) Inflammation, microbiota, and prostate cancer. Eur Urol Focus 2: 374-382.

11. Hayes RB, Pottern LM, Strickler H, Rabkin C, Pope V, et al. (2000) Sexual behavior, STDs, and risks for prostate cancer. Br J Cancer 82: 718-725.

12. Nelson DE, Van Der Pol B, Dong Q, Revanna KV, Fan B, et al. (2010) Characteristic male urine microbiomes associate with asymptomatic sexually transmitted infection. PLoS ONE 5: e14116.

13. Qunfeng Dong, David E Nelson, Evelyn Toh, Lixia Diao, Xiang Gao, et al. (2011) The microbial communities in male first catch urine are highly similar to those in paired urethral swab specimens. PLoS ONE 6: e19709.

14. Celine Cosseau, Deirdre A Devine, Edie Dullaghan, Jennifer L Gardy, Avinash Chikatamarla, et al. (2008) The commensal Streptococcus salivarius K12 downregulates the innate immune responses of human epithelial cells and promotes host-microbe homeostasis. Infect Immun 76: 4163-4175.

15. Aso Y, Akazan H (1992) Prophylactic effect of a Lactobacillus casei preparation on the recurrence of superficial bladder cancer. BLP Study Group. Urol Int 49: 125-129.

16. Nickel JC, Stephens A, Landis JR, Mullins C, van Bokhoven A, et al. (2016) Assessment of the lower urinary tract microbiota during symptom flare in women with urologic chronic pelvic pain syndrome: A MAPP network study. $J$ Urol 195: 356-362.

17. Hilt EE, McKinley K, Pearce MM, Rosenfeld AB, Zilliox MJ, et al. (2014) Urine is not sterile: Use of enhanced urine culture techniques to detect resident bacterial flora in the adult female bladder. J Clin Microbiol 52: 871-876.

18. Meghan M Pearce, Evann E Hilt, Amy B Rosenfeld, Michael J Zilliox, Krystal Thomas-White, et al. (2014) The female urinary microbiome: A comparison of women with and without urgency urinary incontinence. MBio 5: e01283-14. 